\title{
SNObase, a database for S-nitrosation modification
}

\author{
Xu Zhang ${ }^{1,2,3^{*}}$, Bo Huang ${ }^{1 *}$, Lunfeng Zhang ${ }^{1,2}$, Yuying Zhang ${ }^{1,2}$, Yingying Zhao ${ }^{1,2}$, Xiaofei Guo ${ }^{1,2}$, Xinhua Qiao ${ }^{1,2}$, \\ Chang Chen ${ }^{1 凶}$ \\ ${ }^{1}$ National Laboratory of Biomacromolecules, Institute of Biophysics, Chinese Academy of Sciences, Beijing 100101, China \\ ${ }^{2}$ Graduate School of the Chinese Academy of Sciences, Beijing 100049, China \\ ${ }^{3}$ Department of Physiology and Pathophysiology, Key Laboratory of Molecular Cardiovascular Sciences of Education Ministry, \\ Peking University Health Science Center, Beijing 100083, China \\ $\square$ Correspondence: changchen@moon.ibp.ac.cn
}

Received September 1, 2012 Accepted September 12, 2012

\begin{abstract}
S-Nitros(yl)ation is a ubiquitous redox-based posttranslational modification of protein cysteine thiols by nitric oxide or its derivatives, which transduces the bioactivity of nitric oxide (NO) by regulation of protein conformation, activity, stability, localization and protein-protein interactions. These years, more and more $S$-nitrosated proteins were identified in physiological and pathological processes and the number is still growing. Here we developed a database named SNObase (http://www.nitrosation.org), which collected $S$-nitrosation targets extracted from literatures up to June 1st, 2012. SNObase contained 2561 instances, and provided information about $S$-nitrosation targets, sites, biological model, related diseases, trends of S-nitrosation level and effects of $S$-nitrosation on protein function. With SNObase, we did functional analysis for all the SNO targets: In the gene ontology (GO) biological process category, some processes were discovered to be related to S-nitrosation ("response to drug", "regulation of cell motion") besides the previously reported related processes. In the GO cellular component category, cytosol and mitochondrion were both enriched. From the KEGG pathway enrichment results, we found SNO targets were enriched in different diseases, which suggests possible significant roles of $S$-nitrosation in the progress of these diseases. This SNObase means to be a database with precise, comprehensive and easily accessible information, an environment to help researchers integrate data with comparison and
\end{abstract}

relevancy analysis between different groups or works, and also an SNO knowledgebase offering feasibility for systemic and global analysis of S-nitrosation in interdisciplinary studies.

KEYWORDS S-nitrosation, S-nitrosylation, database, S-Nitrosothiol (SNO)

\section{INTRODUCTION}

Protein $S$-nitrosation (commonly refers to $S$-nitrosylation) is a ubiquitous protein post-translational modification (PTM) of free cysteine thiols by nitric oxide (NO) or its derivatives with the formation of S-nitrosothiol (SNO). Protein S-nitrosation is an important pathway for NO bioactivity (Hess et al., 2005) and has received much attention for its effects on protein function and cell signaling through regulating protein activity, localization, stability, protein-protein interactions and other post-translational modifications (Molina y Vedia et al., 1992; Carvalho-Filho et al., 2005; Hara et al., 2005; Huang et al., 2005; Chanvorachote et al., 2006). Many proteins were found to be related to physiological processes (e.g. signal transmission in nervous system (Cheah et al., 2006) and function enhancement in immune system (Saura et al., 1999)) or pathological processes (e.g. neurodegenerative diseases (Chung et al., 2004), diabetes (Carvalho-Filho et al., 2005), cardiovascular disease (Sun et al., 2006), asthma (Atochina-Vasserman et al., 2011)). S-Nitrosothiol is thought to be an important PTM analogous to phosphorylation (Lane et al., 2001).

With the technical development of 2D-electrophoresis, mass spectrometry, protein microarray, etc., high-throughput

\footnotetext{
*These authors contributed equally to the work.
} 
S-nitrosoproteomic data have been continuously generated (Seth and Stamler, 2011), the number of S-nitrosated protein targets has exceeded one thousand and is still growing. Past experience has shown that the construction of the PTM database (like CPLA (Liu et al., 2011), O-GLYCBASE (Hansen et al., 1996), UbiProt (Chernorudskiy et al., 2007), PHOSIDA (Gnad et al., 2007), etc.) can significantly promote the development of the corresponding PTM study. An S-nitrosation database is needed to provide precise, comprehensive and easily accessible information and integrate data to promote comparison and relevancy analysis between different groups or works.

To solve the above mentioned problem, we developed a web-based $S$-nitrosation database named SNObase (www.nitrosation.org). Literatures about single-target S-nitrosation and S-nitrosoproteomic research from PubMed were collected, and information about $S$-nitrosation target, site, biological model, related disease, trends of $S$-nitrosation level and manner of regulation in protein function were extracted manually.

\section{CONSTRUCTION AND CONTENT}

SNObase was developed by open-source softwares. The database system was constructed with MySQL, web query system was written with HTML+php language. The fields of database contains information about Uniprot Accession, Protein Description, Gene Symbol, Species, Model, Pathophysiological (related pathophysiological process), SNO Site, Genous, Throughput, Status, Effects, PubMed PMID, SNObase ID, Alias, Literature title, etc.

We search PubMed in "any field" using the keyword "nitrosation OR nitrosated OR nitrosylation OR nitrosylated"and 3541 results were retrieved (up to June 1st, 2012), 2561 target instances about $S$-nitrosation were collected. For single-target research (low-throughput, LTP), we mapped the SNO target to UniProtKB using protein name and species manually, and related a Uniprot Accession number to each SNO target. For S-nitrosoproteomic research (high-throughput, HTP), the original ID from mass spectrometry-based protein identification in the paper was converted to Uniprot Accession number by DAVID Gene ID Conversion tool (Huang da et al., 2007) or IPI ID mapping tool PICR. The "Gene Symbol" of all the instances were converted from Uniprot Accession number. Alias was manually input as other names of the SNO targets. Information for "Species", "Model", "Related pathophysiological process", "SNO site" was extracted manually. "Genous" describes whether the $S$-nitrosation was endogenous or exogenous. "Throughput" describes whether the instance was acquired from single-target research (LTP) or S-nitrosoproteomic research (HTP). "Status" describes the change of S-nitrosation level in the biological model after treatment. "Effects" describes the regulation of $S$-nitrosation on protein function. "PubMed
PMID" and "Literature title" record corresponding information in PubMed.

\section{USAGE}

Users can access the SNObase at http://www.nitrosation.org. Instances about $S$-nitrosation could be searched in the database with different query, including Description, Gene Symbol, Uniprot Accession, Alias, Literature title, Species, Model, Related pathophysiological process (pathophysiological), SNObase ID.

There are some examples as follows:

1. Users can search if one protein is an SNO target in SNObase with either its "Uniprot Accession", "Gene Symbol", "Description" or "Alias". The most exact results will be displayed when using "Uniprot Accession", for it is species specifically.

2. Users can get information of SNO protein targets in the interested biological model or a pathophysiological process by searching SNObase with "Related pathophysiological process (pathophysiological)".

3. Users can query SNO targets by searching SNObase with key words in "Literature title". It is convenient to get all SNO targets in a known S-nitrosoproteome work.

The query page and the exampled information retrieved are shown as in Fig. 1. In this example, we searched the keyword "diabetes" in the SNObase with the type "Related pathophysiological process", 43 instances were shown in Table 1. In each instance, the "Uniprot Accession" and "PubMed ID" were hyperlinked to the Uniprot protein knowledgebase and PubMed, respectively.

\section{RESULTS AND DISCUSSION}

There are 2561 total instances in SNObase, 505 instances (323 were unique SNO protein targets) were extracted from single target research (LTP) and 2056 instances (1727 were unique SNO protein targets) were extracted from S-nitrosoproteomic research (HTP), as depicted (Fig. 2A). The integrity of SNObase was shown in Fig. 2B. HTP research had relatively more information in the fields of "Gene Symbol", "Species", "Model" and "SNO site", however, LTP research had a relative higher coverage in the fields of "Pathophysiological" and "Effect". Reason of the difference between the appearances is HTP researchers are more inclined to identify SNO targets and sites, but LTP researches are more inclined to identify the function of $S$-nitrosated targets.

Up to date, there were 821 endogenous SNO targets and 1740 exogenous ones (Fig. 2C). Most works showed that SNO increased in their model, a few works studied SNO targets at basal level or de-S-nitrosation (Fig. 2D), which also had important significance. The major species of the research models were human (Homo sapiens), mouse (Mus musculus) and rat (Rattus norvegicus) (Fig. 2E). 


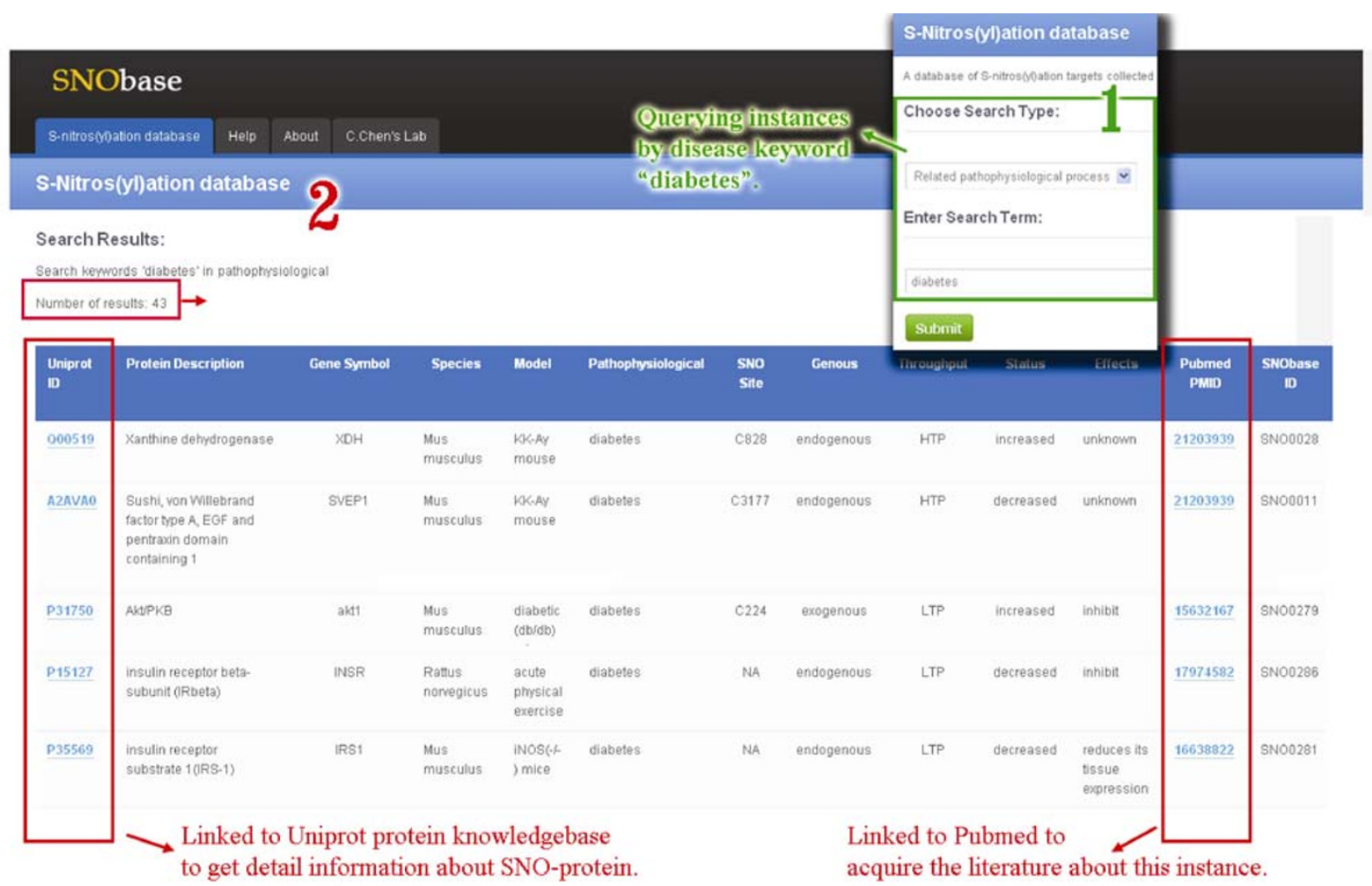

Figure 1. The search option and retrieved page example. Users can query SNObase by searching "diabetes" with the "related pathophysiological process" option (1). The results will be shown in a tabular format (2).

Table 1 The disease related KEGG pathway enriched by SNO target instances in SNObase $(P<0.05$ as significant)

\begin{tabular}{|c|c|c|}
\hline Disease & Related SNO targets in SNObase & $P$-value of KEGG enrichment \\
\hline Prostate cancer & 22 & $2.89 \times 10^{-8}$ \\
\hline Type II diabetes mellitus & 12 & $6.85 \times 10^{-5}$ \\
\hline Arrhythmogenic right ventricular cardiomyopathy (ARVC) & 15 & $1.21 \times 10^{-4}$ \\
\hline Endometrial cancer & 12 & $1.82 \times 10^{-4}$ \\
\hline Dilated cardiomyopathy & 15 & $9.35 \times 10^{-4}$ \\
\hline Glioma & 12 & $1.03 \times 10^{-3}$ \\
\hline Parkinson's disease & 18 & $1.35 \times 10^{-3}$ \\
\hline Hypertrophic cardiomyopathy (HCM) & 14 & $1.35 \times 10^{-3}$ \\
\hline Small cell lung cancer & 13 & $3.68 \times 10^{-3}$ \\
\hline Thyroid cancer & 7 & $6.20 \times 10^{-3}$ \\
\hline Colorectal cancer & 12 & $1.03 \times 10^{-2}$ \\
\hline Bladder cancer & 8 & $1.09 \times 10^{-2}$ \\
\hline Non-small cell lung cancer & 9 & $1.34 \times 10^{-2}$ \\
\hline Alzheimer's disease & 18 & $1.59 \times 10^{-2}$ \\
\hline Pancreatic cancer & 10 & $2.56 \times 10^{-2}$ \\
\hline Chronic myeloid leukemia & 10 & $3.23 \times 10^{-2}$ \\
\hline Amyotrophic lateral sclerosis (ALS) & 8 & $3.57 \times 10^{-2}$ \\
\hline Huntington's disease & 18 & $3.75 \times 10^{-2}$ \\
\hline
\end{tabular}


A

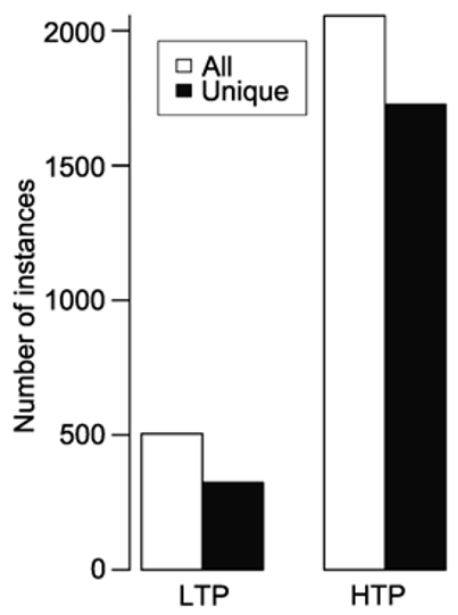

B

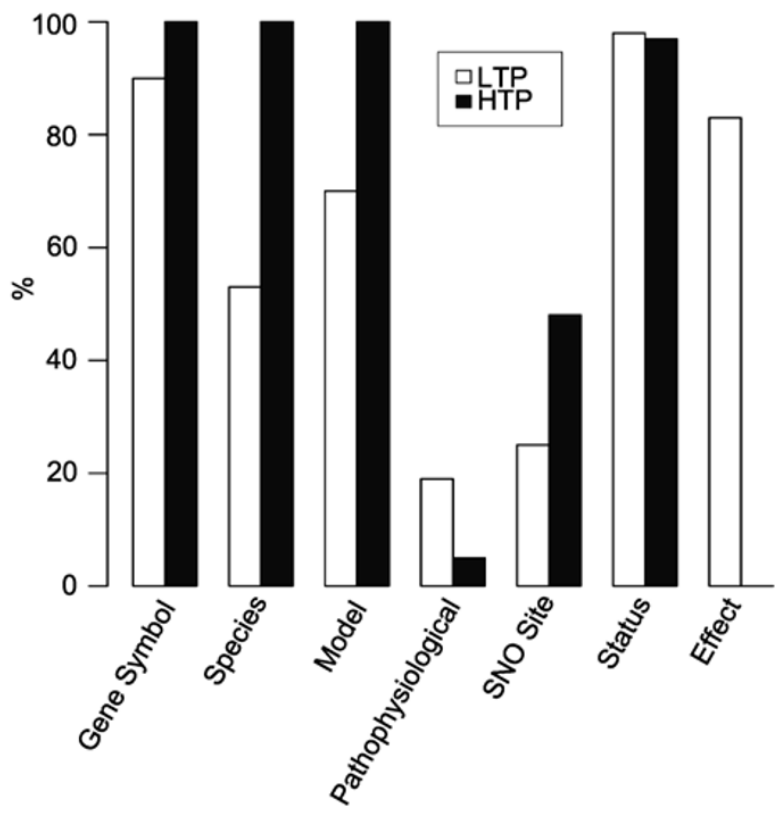

C

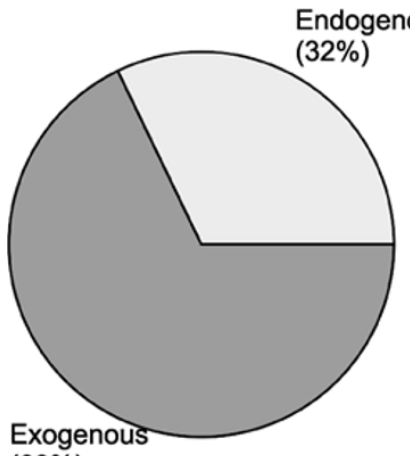
$(68 \%)$
D

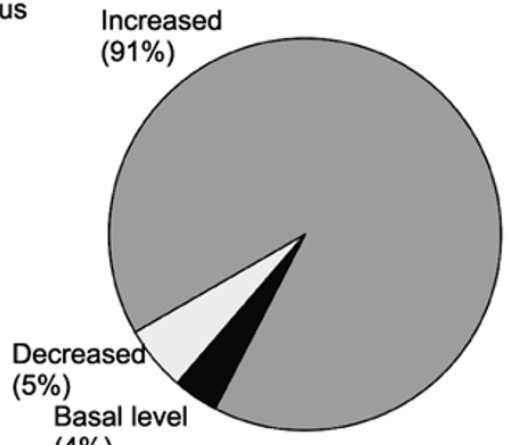

E

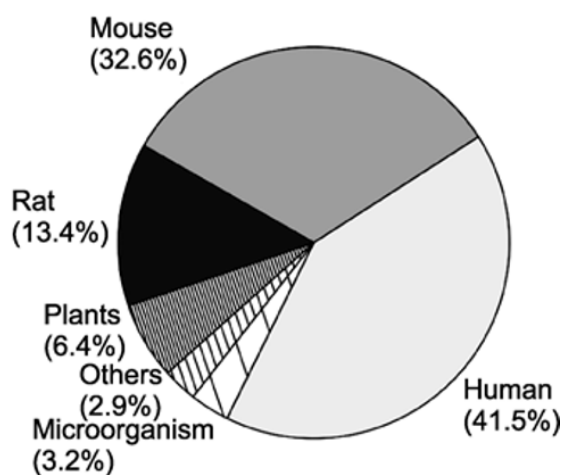

Figure 2. Statistical charts of the instances in SNObase. (A) Number of instances acquired from LTP (single-target experiment) and HTP (S-nitrosoproteomic experiment). (B) The information completeness for some fields in SNObase. (C) Distribution of endogenous SNO and exogenous SNO target instance. (D) Distribution of "Status" of SNO target indicated as increased or decreased. (E) Distribution of "Species" of SNO target instance.

SNObase is very helpful for systematic study of S-nitrosation from global perspective. With SNObase, we did functional analysis for all the SNO targets in the SNObase. Functional Annotation Tool provided by DAVID was used for Gene Ontology (GO) or KEGG pathway enrichment analysis of SNO targets. In the GO biological process category, some processes were enriched by SNO targets in SNObase (Table S1). Many of them are consistent to the previous reports about the SNO related processes such as "glycolysis" (Mohr et al., 1996), "regulation of apoptosis" (Mannick et al., 1999; Nakamura et al., 2010), "protein complex assembly" (Marozkina et al., 2010), and "protein folding" (Uys et al., 2011). However, some processes were seldom related to S-nitrosation (e.g., "response to drug", "regulation of cell mo- tion") in previous studies, which could be new point for S-nitrosation study. In the GO cellular component category, the annotation "cytosol" (especially its sub-annotation "cytoskeleton") was the most enriched one by SNO targets (Table S2). "Mitochondrion" was also enriched, which suggested the occurrence of protein S-nitrosation could be related with the approachability of NO (Broillet, 1999). From the KEGG pathway enrichment results, we found SNO targets enriched in different diseases as listed in Table 1. This suggests $S$-nitrosation may play significant roles in the progress of the disease.

As described above, we constructed an S-nitrosation knowledgebase which covered most of the SNO targets so far. With the help of SNObase, it is efficient for researchers to 
grasp the forefront of $S$-nitrosation and to promote interdisciplinary study. SNObase integrated SNO targets from past works, which offered feasibility for systemic study and global analysis of $S$-nitrosation.

We will constantly improve it in future work. New SNO targets will be added timely. Users are also encouraged to help us to update the database by submitting information.

\section{ACKNOWLEDGEMENTS}

We are grateful to Prof. Taijiao Jiang for the web page refinement. This research was supported by the National Basic Research Program of China (973 Program) (Nos. 2011CB910900, 2012CB911000, 2011CB503900), the National Natural Sciences Foundation of China (Grant No. 31030023) and the Knowledge Innovation Program of the Chinese Academy of Sciences.

\section{ABBREVIATIONS}

GO, gene ontology; HTP, high-throughput; LTP, low-throughput; NO, nitric oxide; PTM, post-translational modification; SNO, S-nitrosothiol

\section{REFERENCES}

Atochina-Vasserman, E.N., Winkler, C., Abramova, H., Schaumann, F., Krug, N., Gow, A.J., Beers, M.F., and Hohlfeld, J.M. (2011). Segmental allergen challenge alters multimeric structure and function of surfactant protein $D$ in humans. Am J Respir Crit Care Med 183, 856-864.

Broillet, M.C. (1999). S-nitrosylation of proteins. Cell Mol Life Sci 55, 1036-1042.

Carvalho-Filho, M.A., Ueno, M., Hirabara, S.M., Seabra, A.B., Carvalheira, J.B., de Oliveira, M.G., Velloso, L.A., Curi, R., and Saad, M.J. (2005). S-nitrosation of the insulin receptor, insulin receptor substrate 1, and protein kinase B/Akt: a novel mechanism of insulin resistance. Diabetes 54, 959-967.

Chanvorachote, P., Nimmannit, U., Stehlik, C., Wang, L., Jiang, B.H., Ongpipatanakul, B., and Rojanasakul, Y. (2006). Nitric oxide regulates cell sensitivity to cisplatin-induced apoptosis through $\mathrm{S}$-nitrosylation and inhibition of Bcl-2 ubiquitination. Cancer Res 66, 6353-6360.

Cheah, J.H., Kim, S.F., Hester, L.D., Clancy, K.W., Patterson, S.E., 3rd, Papadopoulos, V., and Snyder, S.H. (2006). NMDA receptor-nitric oxide transmission mediates neuronal iron homeostasis via the GTPase Dexras1. Neuron 51, 431-440.

Chernorudskiy, A.L., Garcia, A., Eremin, E.V., Shorina, A.S., Kondratieva, E.V., and Gainullin, M.R. (2007). UbiProt: a database of ubiquitylated proteins. BMC Bioinformatics 8, 126.

Chung, K.K., Thomas, B., Li, X., Pletnikova, O., Troncoso, J.C., Marsh, L., Dawson, V.L., and Dawson, T.M. (2004). S-nitrosylation of parkin regulates ubiquitination and compromises parkin's protective function. Science 304, 1328-1331.

Gnad, F., Ren, S., Cox, J., Olsen, J.V., Macek, B., Oroshi, M., and Mann, M. (2007). PHOSIDA (phosphorylation site database): management, structural and evolutionary investigation, and prediction of phosphosites. Genome Biol 8, R250.

Hansen, J.E., Lund, O., Nielsen, J.O., and Brunak, S. (1996). O-GLYCBASE: a revised database of O-glycosylated proteins.
Nucleic Acids Res 24, 248-252.

Hara, M.R., Agrawal, N., Kim, S.F., Cascio, M.B., Fujimuro, M., Ozeki, Y., Takahashi, M., Cheah, J.H., Tankou, S.K., Hester, L.D., et al. (2005). S-nitrosylated GAPDH initiates apoptotic cell death by nuclear translocation following Siah1 binding. Nat Cell Biol 7, 665-674.

Hess, D.T., Matsumoto, A., Kim, S.O., Marshall, H.E., and Stamler, J.S. (2005). Protein S-nitrosylation: purview and parameters. Nat Rev Mol Cell Biol 6, 150-166.

Huang da, W., Sherman, B.T., Tan, Q., Kir, J., Liu, D., Bryant, D., Guo, Y., Stephens, R., Baseler, M.W., Lane, H.C., et al. (2007). DAVID Bioinformatics Resources: expanded annotation database and novel algorithms to better extract biology from large gene lists. Nucleic Acids Res 35, W169-175.

Huang, Y., Man, H.Y., Sekine-Aizawa, Y., Han, Y., Juluri, K., Luo, H., Cheah, J., Lowenstein, C., Huganir, R.L., and Snyder, S.H. (2005). $\mathrm{S}$-nitrosylation of $\mathrm{N}$-ethylmaleimide sensitive factor mediates surface expression of AMPA receptors. Neuron 46, 533-540.

Lane, P., Hao, G., and Gross, S.S. (2001). S-nitrosylation is emerging as a specific and fundamental posttranslational protein modification: head-to-head comparison with O-phosphorylation. Sci STKE 2001, re1.

Liu, Z., Cao, J., Gao, X., Zhou, Y., Wen, L., Yang, X., Yao, X., Ren, J., and Xue, Y. (2011). CPLA 1.0: an integrated database of protein lysine acetylation. Nucleic Acids Res 39, D1029-1034.

Mannick, J.B., Hausladen, A., Liu, L., Hess, D.T., Zeng, M., Miao, Q.X., Kane, L.S., Gow, A.J., and Stamler, J.S. (1999). Fas-induced caspase denitrosylation. Science 284, 651-654.

Marozkina, N.V., Yemen, S., Borowitz, M., Liu, L., Plapp, M., Sun, F., Islam, R., Erdmann-Gilmore, P., Townsend, R.R., Lichti, C.F., et al. (2010). Hsp 70/Hsp 90 organizing protein as a nitrosylation target in cystic fibrosis therapy. Proc Natl Acad Sci U S A 107, 11393-11398.

Mohr, S., Stamler, J.S., and Brune, B. (1996). Posttranslational modification of glyceraldehyde-3-phosphate dehydrogenase by S-nitrosylation and subsequent $\mathrm{NADH}$ attachment. J Biol Chem 271, 4209-4214.

Molina y Vedia, L., McDonald, B., Reep, B., Brune, B., Di Silvio, M., Billiar, T.R., and Lapetina, E.G. (1992). Nitric oxide-induced S-nitrosylation of glyceraldehyde-3-phosphate dehydrogenase inhibits enzymatic activity and increases endogenous ADP-ribosylation. J Biol Chem 267, 24929-24932.

Nakamura, T., Wang, L., Wong, C.C., Scott, F.L., Eckelman, B.P., Han, X., Tzitzilonis, C., Meng, F., Gu, Z., Holland, E.A., et al. (2010). Transnitrosylation of XIAP regulates caspase-dependent neuronal cell death. Mol Cell 39, 184-195.

Saura, M., Zaragoza, C., McMillan, A., Quick, R.A., Hohenadl, C., Lowenstein, J.M., and Lowenstein, C.J. (1999). An antiviral mechanism of nitric oxide: inhibition of a viral protease. Immunity 10, 21-28.

Seth, D., and Stamler, J.S. (2011). The SNO-proteome: causation and classifications. Curr Opin Chem Biol 15, 129-136.

Sun, J., Picht, E., Ginsburg, K.S., Bers, D.M., Steenbergen, C., and Murphy, E. (2006). Hypercontractile female hearts exhibit increased S-nitrosylation of the L-type Ca2+ channel alpha1 subunit and reduced ischemia/reperfusion injury. Circ Res 98, 403-411.

Uys, J.D., Xiong, Y., and Townsend, D.M. (2011). Nitrosative stress-induced S-glutathionylation of protein disulfide isomerase. Methods Enzymol 490, 321-332. 